\title{
Die Kosten- und Leistungsrechnung - Ein Blick auf mehr als 70 Jahre Vergangenheit und eine spannende Zukunft
}
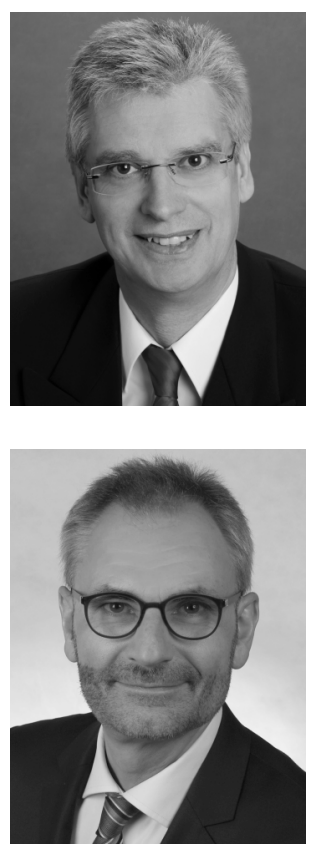

\section{Dieter Pfaff und Ernst Troßmann}

Kosten- und Leistungsrechnung; Internes Rechnungswesen; Entwicklungsgeschichte; Zukunftsaspekte; methodische Entwicklungen

cost accounting; managerial accounting; historical evolution; future developments; methodological aspects.

Im vorliegenden Beitrag werden die Entwicklungslinien der Kostenund Leistungsrechnung bis zum heutigen Stand nachgezeichnet. Davon ausgehend werden zudem die Themen und Ansätze festgemacht, die den derzeitigen Stand, die aktuelle Diskussion und die vermuteten Entwicklungen der nächsten Jahre und Jahrzehnte bestimmen.

This essay traces the lines of development of cost accounting to the present state. Based on the historical evidence we carve out the topics and approaches that determine the current state and the expected developments in the next years and decades.

\section{Die Kosten- und Leistungsrechnung - langweiliges Pflichtteil- Erbe einer traditionellen Betriebswirtschaftslehre?}

Die Kosten- und Leistungsrechnung gilt als eine der ältesten Teildisziplinen der Betriebswirtschaftslehre. Und sie ist zugleich eines der wenigen betriebswirtschaftlichen Gebiete, die im deutschsprachigen Raum am sorgfältigsten ausgearbeitet wurden und die deshalb von hier ausgehend nahezu weltweit Nachahmer gefunden haben. Anders als im Bereich der Buchführung, wo bis heute die typischen Fachbezeichnungen an deren italienischen Ursprung erinnern, hat sich allerdings die Herkunft im Bereich der Kosten- und Leistungsrechnung in den äußeren Bezeichnungen nicht niedergeschlagen.

Die Kosten- und Leistungsrechnung zeigt bis in die heutige Zeit hinein eine erstaunliche Konsequenz in ihrer Entwicklung. Erstaunlich ist dies deshalb, weil die Einzelbeiträge, die über mehr als 100 Jahre hinweg dazu geliefert wurden - die Ursprünge liegen sogar deutlich früher und gründen in der Kalkulation des Handels und der Manufaktur im ausgehenden 18. Jahrhundert (vgl. Dorn 1961, S. 23-26) -, bei ihrer Entstehung durchaus eher zufälligen und singulären Charakter hatten. Dies hat in einer umfangreichen Analyse jüngst der Arbeitskreis Internes Rechnungswesen der Schmalenbach-Gesellschaft festgestellt. Er hat in einer aktuellen Veröffentlichung unter dem Titel „Säulen der Kostenrechnung “ markante Originaltexte zur kostenrechnerischen Entwicklung aus den vergangenen 100 Jahren zusammengestellt und kommentiert. Basierend auf der Analyse dieser, aber auch zahlrei- 
cher anderer Quellen sowie eigener Erkenntnisse zeichnen die beiden Autoren des vorliegenden Beitrags - beide zugleich Mitglieder des genannten Arbeitskreises - hier die Entwicklungslinien der Kosten- und Leistungsrechnung bis zum heutigen Stand nach und machen daran die Themen und Ansätze fest, die den derzeitigen Stand, die aktuelle Diskussion und die vermuteten Entwicklungen der nächsten Jahre bestimmen. Der 70. Geburtstag der Zeitschrift „Die Unternehmung“ ist ein guter Anlass für diesen Rück- und Vorblick, wenn auch im Bereich der Kosten- und Leistungsrechnung zumindest im Rückblick zu den 70 Jahren noch etwas dazugelegt werden muss.

In der angesprochenen Entwicklung der Kosten- und Leistungsrechnung bis zum heutigen methodischen Stand lassen sich grob folgende Phasen unterscheiden:

Erste Phase:

Zweite Phase:

Dritte Phase:

Vierte Phase:

Fünfte Phase:

Sechste Phase:

Siebte Phase:

Entwicklung der grundlegenden Rechenstrukturen

Verfeinerung der Rechenstrukturen in den Einzelmethoden

Inhaltliche Diskussion um die „richtige“ Kostenzurechnung

Integration zu Gesamtsystemen der Kosten- und Leistungsrechnung

Methoden zur differenzierten entscheidungsorientierten Bewertung

Weiterentwicklung zu Kostenmanagement-Methoden

Genauere Differenzierung der Kosten- und Leistungsrechnung nach angestrebten Führungszielen

Diese Phasen lassen sich über die langen Jahre der Publikationen, über eine endlose Reihe von Zeitschriften-Aufsätzen und einige Regalmeter Bücher nachverfolgen, sind aber in ihrem Kern dennoch markant charakterisierbar. Dabei sind die Phasen nicht ganz eindeutig zeitlich abgegrenzt, sondern überlappen sich oft - bisweilen findet sich auch zu einem späteren Zeitpunkt ein besonders gelungen und pointiert geschriebener Endpunkt einer mehrjährigen Diskussion. Abbildung 1 zeigt die Phasenabfolge schematisch im Überblick. Die vollständige Nennung aller Autoren ist schon aus Platzgründen nicht bezweckt.
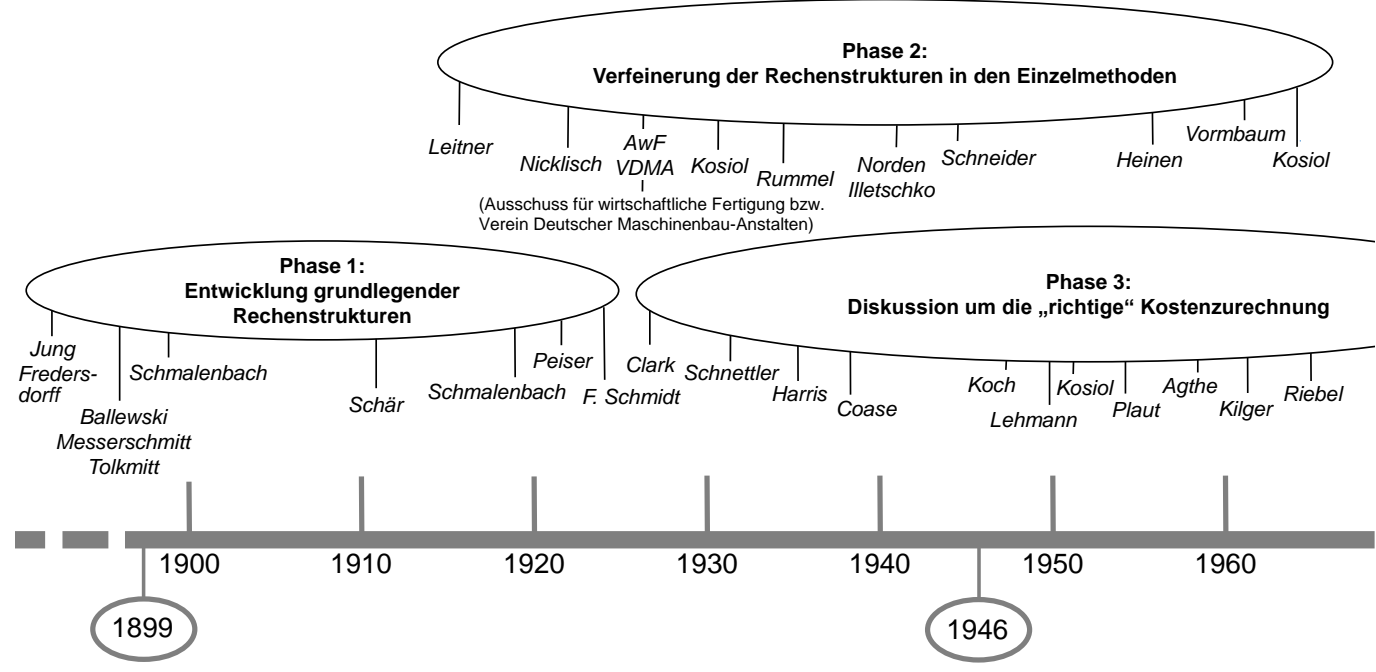

Abbildung 1: Entwicklungsphasen der Kosten- und Leistungsrechnung 


\section{Herausbilden der Kostenrechnung als Kern eines eigenen Rechensystems}

\subsection{Erste Phase: Entwicklung der grundlegenden Rechenstrukturen}

Phase 1 ist die historisch älteste Phase. Hier finden sich die ersten Überlegungen zu Rechenmethoden und Vorgehensweisen der Kostenrechnung, dies in einem Umfeld, das noch wenig „gesichertes Wissen“ im kaufmännischen Bereich insgesamt vorweisen kann. Der Ursprung liegt dabei in der Kalkulation (vgl. z. B. Jung 1786, Fredersdorff 1802, Ballewski 1880, Messerschmitt 1884 und Tolkmitt 1894). Obwohl es sich bei den Autoren dieser Zeit fast ausnahmslos um Praktiker handelt, ist Ende des 19. Jahrhunderts ein einfaches Kalkulationswesen in der Praxis noch kaum verbreitet. Noch weniger verbreitet ist der Gedanke einer zeitraumbezogenen Rechnung. Mit der rasanten technischen Entwicklung zum Großunternehmen wächst zu Beginn des 20. Jahrhunderts das Interesse an verbesserten Kalkulationsverfahren und an einer Abbildung innerbetrieblicher Vorgänge ganz allgemein. Die Impulse gehen sowohl von der Praxis als auch den Handelshochschulen aus. Dabei entsteht die Einteilung in Kostenarten-, -stellen-, -trägerrechnung und Betriebsergebnisrechnung - im Wesentlichen genauso, wie wir sie heute für selbstverständlich halten.

Grundlegend ist vor allem der Artikel „Buchführung und Kalkulation im Fabrikgeschäft" von Eugen Schmalenbach, bereits 1899 erschienen. Bemerkenswert ist, dass Schmalenbach schon dort das Zuschlagskalkulationsprinzip vorstellt, ohne dass wir heute wissen, ob es sein Vorschlag war oder ob er nur den bereits bestehenden Handelsbrauch beschrieben hat. Ironie der Geschichte ist, dass das nationalsozialistisch regierte Deutschland fast 40 Jahre danach die Vorschläge des gleichen Schmalenbachs im Reichswirtschaftsministerium verwendet, den es aus mehreren Gründen von seinem Kölner Lehrstuhl vertrieben hatte. Jedenfalls aber haben durch die Schmalenbach'schen Vorlagen und einen darauf aufbauenden ministerialen Erlass (siehe auch die Leitsätze für die Preisermittlung aufgrund der Selbstkosten bei Leistungen für öffentliche Auftraggeber - LSÖ) das Zuschlagskalkulationsschema wie auch die weiteren methodischen Strukturen der Kosten-

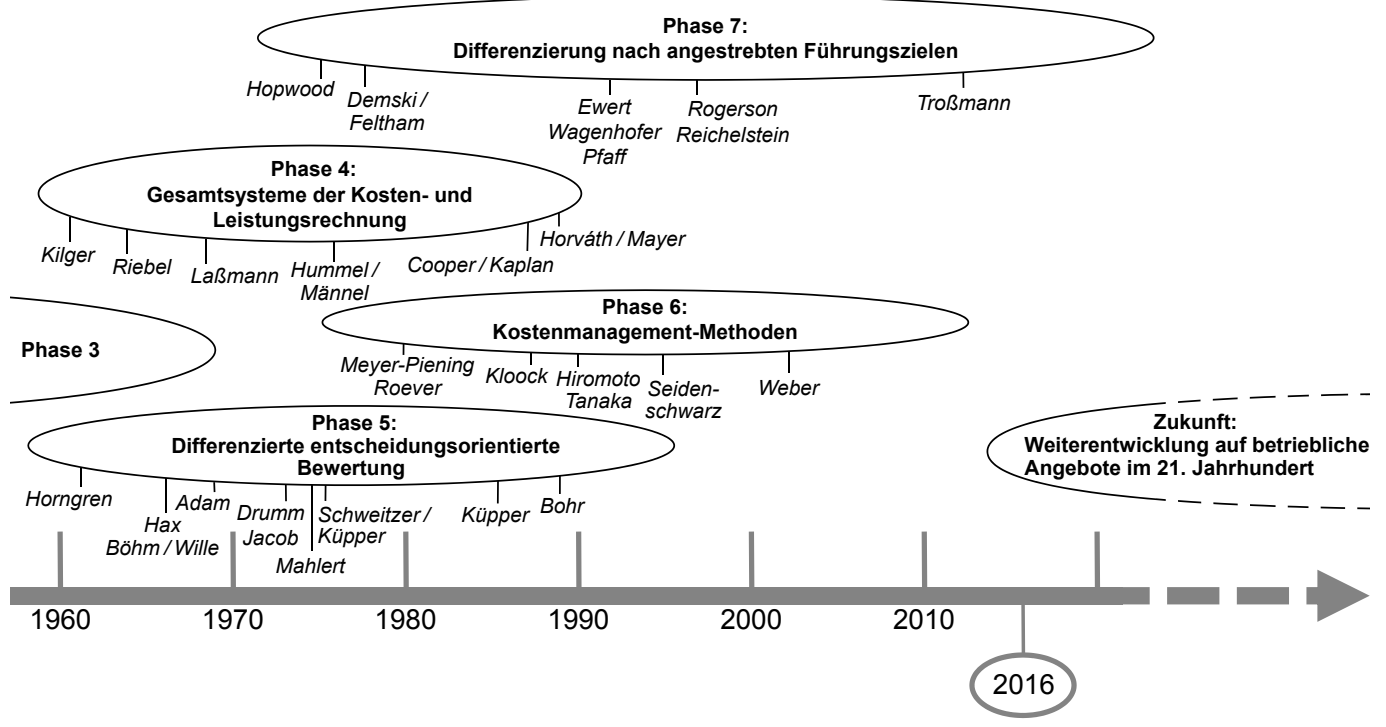


rechnung in Deutschland eine nahezu flächendeckende Verbreitung gefunden. Hintergrund dieser Entwicklung ist die - besonders in Kriegsjahren - ständig zunehmende Vergabe von staatlichen Aufträgen an die private Wirtschaft und die damit verbundene Notwendigkeit der Preissetzung. Nach dem Krieg werden die LSÖ durch die Leitsätze für die Preisermittlung aufgrund von Selbstkosten (LSP) abgelöst, die Art des Kalkulationsverfahrens bleibt jedoch weitgehend unverändert. Einige Autoren glauben (so Riebel 1956, S. 278), dass der von der öffentlichen Auftragsvergabe ausgehende Zwang zur Vollkostenrechnung für den großen Verbreitungsgrad dieses Systems mitverantwortlich sei. Insgesamt jedenfalls sind Rechenstrukturen, Tabellenaufbau und Rechenabfolgen ein halbes Jahrhundert nach den ersten Schmalenbach'schen Veröffentlichungen im deutschsprachigen Raum ziemlich einheitlich. Neben dem markanten Zuschlagskalkulationsschema gilt das für eine ganze Reihe von kostenrechnerischen Detailmethoden, seien es Verbrauchs- und Bestandsberechnungen, innerbetriebliche Leistungsverrechnungen oder eben auch die Periodenergebnisrechnung.

Selbstverständlich fallen in die Phase 1 weitere Veröffentlichungen, so etwa aus Schweizer Sicht die von Johann Friedrich Schär und Karl Käfer. Johann Friedrich Schär wurde 1903 auf den Lehrstuhl für Handelswissenschaft der Universität Zürich berufen; zuvor war er Präsident des Verbands Schweizer Konsumvereine, heute Coop. Von 1906 bis 1919 war Schär Professor an der Handelshochschule Berlin. Wichtige Beiträge Schärs finden sich zur Allgemeinen Handelsbetriebslehre (1911), zur Buchhaltung und Bilanz, aber auch zu Kosten, etwa seinen Überlegungen zum „toten Punkt“ (Schär 1911, S. 134 f.; heute würde man vom Break-even-Punkt sprechen). Karl Käfer entwickelte 1947 einen Kontenrahmen (unter Einbezug der Betriebsbuchhaltung), dessen Einfluss gerade auf kleinere und mittelständische Unternehmen bis heute sichtbar ist. Käfer gilt als prominentester Vertreter der Schweizer Betriebswirtschaftslehre seiner Zeit. Er gründete die Zeitschrift Die Unternehmung vor 70 Jahren und publizierte mehr als 100 Schriften, die besonders in der Schweiz, aber auch in Japan deutlichen Einfluss auf Wissenschaft und Praxis hatten. Aus kostenrechnerischer Sicht ist besonders „seine“ Standardkostenrechnung zu betonen (vgl. Käfer 1955).

$\mathrm{Zu}$ nennen ist weiterhin Fritz Schmidt, der die Frage, ob die Anschaffungs- bzw. Herstellungskosten (historische Werte) oder die Wiederbeschaffungskosten (Tageswerte) die Grundlage für die Kostenrechnung sein sollten, maßgeblich beeinflusste. Geprägt durch die Markttheorie seiner Zeit war er der Überzeugung, dass „nur die Kostenrechnung mit dem Werte des Umsatztages ... das Gleichgewicht in der Wirtschaft dauernd" verbürgen könne (Schmidt 1923, S. 100). Vereinzelt reichen Beiträge der Kategorie aus Phase 1 in Details bis in die 1950er Jahre hinein, die wesentlichen Vorgaben stammen jedenfalls aus dem späten 19. sowie dem frühen 20. Jahrhundert.

\subsection{Zweite Phase: Verfeinerung der Rechenstrukturen in den Einzelmethoden}

Damit ist der Übergang zur zweiten Phase bereits angesprochen. Hier steht die Verfeinerung der Methoden im Vordergrund. Man beschäftigt sich mit den verschiedenen Möglichkeiten und zugehörigen Anwendungsbereichen der Verrechnung von Sekundärleistungen, also den Umlagemethoden von Vor- auf End- und andere Kostenstellen, ein in der Vor-EDV-Zeit durchaus lohnendes Thema. Entsprechendes gilt für allerlei Feinheiten der Schnittstellen zwischen der Buchführung und der Kostenrechnung, was in der Vor-SAPZeit zu Diskussionen über Ein- und Zweikreissysteme der Buchführung führt. Solche The- 
men verschwinden im Laufe der Zeit wieder von der erkennbaren Oberfläche der Diskussion, waren aber keineswegs uninteressant oder gar überflüssig, haben sie doch wichtige Grundlagen für spätere Software-Lösungen gelegt. Was ebenfalls in diese Kategorie der Feinentwicklung gehört, sind auch verschiedene Ausgestaltungsformen der Kalkulation. So findet man in einer zusammenfassenden Publikation von Vormbaum (1966) sehr ausdifferenzierte Formen der Zuschlagskalkulation, ebenso wie in den Kostenrechnungsbeiträgen von Erich Kosiol, der in der ihm eigenen Systematisierungssorgfalt u. a. die mehrfache mehrstufige Zuschlagsrechnung als differenzierteste Form von anderen Möglichkeiten der Zuschlagskalkulation unterscheidet (vgl. erstmals Kosiol 1964). Eine konsequente Umsetzung der in solchen Publikationen der damaligen Zeit aufgeführten Zuschlagskalkulationsformen würde manche Prozesskostenkalkulation des Jahres 2016 als harmlos und verkürzt erscheinen lassen.

\section{Inhaltliches Ringen um eine richtige Kosten- und Leistungsrechnung als Gesamtwerk}

\subsection{Dritte Phase: Inhaltliche Diskussion um die „richtige“ Kostenzurechnung}

Die dritte Phase der kostenrechnerischen Entwicklung setzt eine inhaltliche Diskussion in Gang, die letztlich bis heute andauert, wenn auch unterdessen die ideologischen Komponenten der Argumente als geklärt und überwunden gelten können: Es handelt sich um die Frage nach Voll- oder Teilkosten. Aufgeworfen auch durch Aufsätze amerikanischer Autoren (so etwa Clark 1923 oder Coase 1938, vgl. dazu den auszugsweisen Nachdruck in den „Säulen der Kostenrechnung“, herausgegeben vom Arbeitskreis Internes Rechnungswesen 2017, Kapitel III), hat die Teilkostenidee ihre konsequentesten Befürworter als auch Gegner wohl bei den deutschsprachigen Fachwissenschaftlern gefunden. Die Auseinandersetzung damit überspannt kriegsbedingt einen großen Zeitraum, der in Europa bis über die 1950er Jahre hinweg in die 1960er Jahre hineinreicht. Dabei, das kann im emotionsfreien Rückblick festgestellt werden, haben die Befürworter der Teilkostenrechnung in der Fachwissenschaft und entsprechend in der Fachliteratur weiträumig das Feld für sich gewonnen, während parallel dazu die Umsetzung in der betrieblichen Praxis weit weniger konsequent und schon gar nicht begeistert vollzogen wurde.

Dass dies so ist, hängt vor allem an einer nicht konsequent genug geführten Zweckdiskussion („different costs for different purposes“ - Clark 1923). Während Teilkostenrechner in der Regel die Entscheidungsfundierung als Hauptzweck einer laufenden Kostenrechnung annehmen, will die steuerungsorientierte Praxis die informatorische Voraussetzung für eine operative Vorgabe und Kontrolle schaffen (zu Einzelheiten vgl. Pfaff/Weber 1998). Dabei ist es der Praxis wichtig, dass am Ende alle Kosten auf Verantwortungs- und Produktbereiche verteilt sind (Teilkostenrechner würden demgegenüber von einer unzulässigen „Schlüsselung“ sprechen). Aus Sicht der Praxis sind die zugerechneten Fix- und anderen Gemeinkosten aber nichts anderes als „Soll-Deckungsbeiträge“, welche die steuernden Einheiten in ihrer Planung berücksichtigen (siehe auch weiter unten die Ausführungen zur Verhaltenssteuerung der Kostenrechnung). Dies betrifft vor allem auch die leidige Zurechnung von Overheadkosten zentraler Bereiche. Soll-Deckungsbeiträge finden sich übrigens auch bei Riebel, der mit seinem System der relativen Einzel- und Deckungsbeitragsrechnung (Riebel 1964) als konsequentester Teilkostenrechner zu bezeichnen ist. Allerdings muss eingeräumt werden, dass die (vollkostenrechnende) Praxis die Soll-Deckungsbeiträge 
selten als solche explizit ausweist, was zu Missverständnissen (und eben leidenschaftlichen Diskussionen) in Wissenschaft und Praxis führen kann.

Auf derselben Argumentation fußt letztlich auch die Unterscheidung sogenannter ,absoluter“ und „kurzfristiger“ gegenüber „langfristiger“ Preisuntergrenzen, die sich so schnell durchsetzt, dass die eigentlichen Urheber heute nicht mehr identifizierbar sind. Die Wirkung ist (und zwar bis heute), dass die Teilkostenrechner die ihnen wichtigen variablen Stückkosten in der „kurzfristigen“ Preisuntergrenze wiederfinden, die Vollkostenrechner dagegen (,irgendwie zugeordnete“) anteilige Fixkosten als Zusatzsummand in der „langfristigen " Preisuntergrenze unterbringen können. Dieser Formelkompromiss erlaubt eine Koexistenz von Voll- und Teilkostenrechnern, und dies über viele Jahre hinweg, wie einschlägige Kostenrechnungslehrbücher bis heute zeigen. Tiefgründig geht es aber nicht um die Frage „kurzfristig“ oder „langfristig“, sondern um die Frage, welche Kosten in einem Entscheidungszeitpunkt noch disponibel und welche bereits versunken (sunk) sind. $\mathrm{Zu}$ einem Zeitpunkt, in dem bestimmte mengenfixe Kosten erst noch aufgebaut werden müssen, kann man dann einerseits bei gegebenem bzw. geplantem Preis nach der kritischen Menge (der Break-even-Menge oder dem „toten“ Punkt in der Terminologie Schärs) fragen, aber auch bei gegebener Menge nach dem kritischen Preis (dem Break-even-Preis, dem unteren Grenzpreis), der erreicht werden muss, um Gewinn erwirtschaften zu können. Break-even-Mengen und Preisuntergrenzen sind somit Indikatoren, die Aufschluss geben, ob ein bestimmtes Geschäft überhaupt lohnt. Bei der Frage nach dem kritischen (Durchschnitts-) Preis bei noch aufzubauenden Fixkosten müssen diese jedoch zwangsläufig durch die geplante Menge dividiert (oder geschlüsselt) werden, um einen entsprechenden Indikator zu erhalten. Die Ablehnung einer solchen Division durch konsequente Teilkostenrechner löst sich aus heutiger Sicht in einer übergeordneten Betrachtung auf: Beim angesprochenen Fixkostenaufbau handelt es sich genau genommen um eine produktartbezogene Investitionsentscheidung, für die ohnehin (übereinstimmend) der Übergang zu einer entsprechenden Investitionsrechnung empfohlen wird (vgl. dazu Kapitel 5). Jene ist aber in jedem Fall nicht stückbezogen, sondern betrifft die Gesamtproduktionsmenge.

Die Definition (mengen-)variabler Kosten hat über die Frage der „richtigen“ Preisuntergrenze hinaus indessen noch weitaus bedeutendere Konsequenzen für die Entwicklung des Gebietes: Zum einen handelt es sich um die angesichts der inhaltlichen Diskussion nahezu unbeachtete Übereinstimmung darin, dass nicht Preise, sondern Preis(unter)grenzen kalkuliert werden. Zum anderen hat die mit der Einführung variabler Kosten zwangsläufig zu definierende Größe des Deckungsbeitrags die Konsequenz, dass die Kosten und ihr Gegenstück, nämlich die Leistungen, nunmehr zu einer sinnvollerweise gemeinsam zu betrachtenden Einheit werden: Die Kostenrechnung wird damit zu der auch heute noch so bezeichneten Kosten- und Leistungsrechnung.

Die Diskussion um die „richtige“ Kostenzurechnung verästelt sich in den 1960er Jahren zunehmend weiter. Sie bringt eine Reihe von Unterscheidungen und Begriffen hervor, von denen viele heute wieder vergessen sind. Eine Hauptunterscheidung freilich erscheint besonders zentral, sie ist auch bis heute geblieben: die Einteilung in Einzel- sowie Gemeinkosten einerseits und variable sowie fixe Kosten andererseits. Diese Unterscheidung gewinnt zunächst vor allem dadurch besondere Aufmerksamkeit, weil sich für ihre vorherrschende Verwendung jeweils markante Fachvertreter einsetzen und sich im Streit um die richtige Vorgehensweise das Lager der Teilkostenrechner in den 1960er und 1970er Jahren aufspaltet. Allerdings sind es sehr unterschiedliche Gruppen: Auf der einen Seite sammeln 
sich mit Wolfgang Kilger (1961) und seiner „Grenzplankostenrechnung“ zahlreiche Professorenkollegen als Vertreter einer Rechnung mit variablen Kosten. Auf der anderen Seite steht Paul Riebel (1964) als Vertreter einer strikt an (relativen) Einzelkosten orientierten Rechnung nahezu alleine. Aus heutiger Sicht ist bemerkenswert, dass beide letztlich nur die „richtigen“ Teilkosten präziser fassen wollten und dabei unterschiedliche Ausgangsprobleme, Erklärungsstränge und Argumentationsmuster zugrunde legten. Während Riebel ohne Zweifel der konsequentere Logiker ist, dafür aber gerne die herrschende Lehre und bereits eingeführte Definitionen ignoriert, ist Kilger oft bereits in der Begriffsfassung allzu bereit, später vielleicht notwendige Umsetzungskompromisse gleich vorweg zu nehmen, was seinen Konzepten bisweilen sehr angreifbare, schwammige Abgrenzungen beschert. Beide haben unverzichtbare Elemente zur modernen Konzeption der Teilkostenrechnung beigetragen. Die Auseinandersetzung zwischen ihnen und insbesondere die Riebels mit zahlreichen anderen Kostenrechnungs-Professoren mag dabei zu zusätzlicher Präzisionsschärfe beigetragen haben.

Aus heutiger Sicht ist nicht ausgeschlossen, dass das, was nach der Kilger'schen Einteilung die eigentlichen variablen Kosten - unter Vermeidung aller hilfsweisen Zurechnungen - sind, durchaus mit dem übereinstimmt, was Riebel als „automatisch“ sich verändernde „Leistungskosten“ anspricht.

\subsection{Vierte Phase: Integration zu Gesamtsystemen der Kosten- und Leistungsrechnung}

Die in Phase 3 entbrannte Diskussion geht einher mit einer letztlich davon unabhängigen Entwicklung: der Konzeption ganzer Rechensysteme, also einer Struktur für die Kostenund Leistungsrechnung des gesamten Betriebes. Hier geht es einerseits um das Ineinandergreifen der verschiedenen Teilschritte der Kosten- und Leistungsrechnung von der Kostenartenerfassung bis zur Präsentation des Kosten- und Deckungsbeitragsplans sowie der Vorlage einer Betriebsergebnisrechnung. Andererseits rückt auch die inhaltlich sinnvolle Gliederung von Rechenschritten sowie die Rechen- und Darstellungsstruktur in den Vordergrund. Während in Phase 1 und in Phase 2 eher isolierte Strukturelemente, wie der Betriebsabrechnungsbogen, das Betriebsergebniskonto, das Kalkulationsschema usw., diskutiert werden, geht es jetzt um ein zusammengehörendes Struktursystem für die gesamte Kostenrechnung. Erstaunlicherweise entwickeln sich die bedeutenden Vorschläge parallel zur Diskussion um eine Voll- oder Teilkostenrechnung und sind auch mit den Namen der dortigen Kontrahenten verbunden, obwohl das eine mit dem anderen genau genommen nichts zu tun hat.

Das System Kilgers (1961) ist von allen Vorschlägen das erfolgreichste, an der Verbreitung und der Umsetzungseinfachheit gemessen. Grund dafür ist die enge Zusammenarbeit Kilgers mit Plaut, einem frühen Anbieter von Kostenrechnungssoftware. Später gehen dessen EDV-Lösungen in das Kostenrechnungssystem von SAP über, dem heutigen Weltmarktführer. Das Prinzip des Kilger'schen Systems beruht auf einer Kostenstellengliederung des gesamten Betriebs mit durchweg einfach-linearen Kostenfunktionen. Alle entsprechenden Rechnungen bestehen daher letztlich in einer Addition separabler Kostenfunktionen (vgl. Troßmann 1992). Aus der gleichen Zeit stammt das von Laßmann (1968) vorgeschlagene System der sogenannten „Einflussgrößenrechnung“. Es folgt dem gleichen Prinzip einer Zerlegung in linear-additive Kostenkomponenten. Die Besonderheit bei Laßmann liegt in der äußerlichen Formalstruktur auf Basis einer speziellen Input-Output-Matrix. Anwen- 
dungen findet dieses System vor allem im Montanbereich. Letzteres erklärt auch den geringen Verbreitungsgrad.

Bemerkenswert, weil vielfach gar nicht unmittelbar in seiner Bedeutung wahrgenommen, ist das Struktursystem, mit dem Paul Riebel (1964) seine Version der Deckungsbeitragsrechnung umsetzt. Er entwickelt Gliederungsprinzipien von Bezugsobjektshierarchien, die letztlich alternative Aufbaustrukturen von Deckungsbeitragssystemen begründen (vgl. Riebel 1964). Dies ist für die entscheidungsorientierte Gliederung der Kosten und die Berechnung von Deckungsbeiträgen für betriebliche Teilbereiche von zentraler Bedeutung. Es ermöglicht insbesondere die Berechnung und Zuordnung passender Deckungsbeiträge zu Verantwortungsbereichen. Und es ist völlig unabhängig von dem verfolgten Definitionskonzept der verwendeten Teilkosten, liegt also außerhalb des Streites innerhalb der Teilkostenrechner.

In die Phase der Entwicklung von Kostenrechnungsstrukturen gehört auch die Systematik der Behandlung von Kuppelproduktionen, einem Teilgebiet der Kosten- und Leistungsrechnung, das bis heute weitgehend ein Schattendasein führt. Aus verschiedenen Gründen ist es aber von höchst aktueller Bedeutung, wie sich insbesondere in unserem Blick auf $\mathrm{Zu}$ kunftsaspekte des internen Rechnungswesens zeigt. Der Kerngedanke, auch wieder auf Riebel zurückgehend, liegt in der Definition von Produktpäckchen. In ihnen werden immer solche Mengen der beteiligten Kuppelprodukte zusammengefasst, wie es den Kuppelverhältnissen entspricht. So entstehen tatsächlich disponierbare Entscheidungsgrößen. Die korrekte rechnerische Behandlung, neben der definitorischen Idee die zweite wesentliche Strukturleistung, wurde 1973 vor allem von Kruschwitz beigesteuert (vgl. Kruschwitz 1973, vgl. ferner Troßmann/Baumeister/Werkmeister 2013).

Zur Phase der Kostenrechnungssysteme gehört genau genommen auch die Prozesskostenrechnung, wenngleich sie zeitlich mit dem Ende der 1980er Jahre eher als Nachzügler der prinzipiell früher liegenden Phase 4 anzusehen ist. Grundlegend ist die Annahme, dass Bezugsobjekte (Produkte, Kunden, Lieferanten, Beschaffungswege usw.) Kosten verursachen, indem sie Aktivitäten, Teil- oder Hauptprozesse beanspruchen, die ihrerseits wiederum Ressourcen verzehren. Die Prozesskostenrechnung steht damit in der Tradition des "Verursachungsprinzips“ (und nicht des „Marginalprinzips“), empfiehlt jedoch ein Rechensystem, das sich auf den ersten Blick deutlich von den anderen Kostenrechnungssystemen unterscheidet, inhaltlich aber weitgehend mit dem System der Grenzplankostenrechnung Kilgers übereinstimmt (vgl. Franz 1990). Dies erstaunt zunächst, weil die ersten und auch bis heute die häufigsten Anwendungen der Prozesskostenrechnung inhaltlich regelmäßig als Vollkostenrechnung ausgebildet sind - dies ist aber nach der Konstruktionsidee eben keineswegs zwingend. Für die typischen Elemente des Prozesskostenrechnungssystems haben sich, vermutlich in Verkennung der starken Analogie und weil die originären Beiträge aus den USA kommen (vgl. z.B. Cooper/Kaplan 1988, Cooper 1989), völlig andere Bezeichnungen durchgesetzt: Cost Driver statt Kostenbestimmungsgröße, Cost-DriverRate statt Gemeinkostenzuschlag, Prozesskosten statt speziell definierte Teilsumme von Kostenstellenkosten usw. Die Prozesskostenrechnung wird deshalb auch als ein Kostenrechnungssystem interpretiert, das besonders auf Dienstleistungsprozesse und betriebliche Sekundärleistungen hin orientiert ist. Verbunden damit hat sich im kollektiven Kostenrechnungsbewusstsein die Produktion immaterieller Güter als Schwergewicht der inhaltlichen Zuordnung festgesetzt. Dies ist zwar methodisch kaum gerechtfertigt, tatsächlich hat es aber eine positive Umsetzungswirkung entfaltet. Sie hat nämlich dazu geführt, dass in 
betrieblichen Bereichen (fallweise) kostenrechnerische Analysen durchgeführt worden sind, die vordem als schwer bearbeitbar angesehen und deshalb kostenrechnerisch übergangen wurden. In vielen Fällen hat dies die kostenrechnerische Basis zahlreicher Anwendungen, neben der Kalkulation beispielsweise auch die Kostenbudgetierung oder die innerbetriebliche Leistungsverrechnung, deutlich verbessert.

\subsection{Fünfte Phase: Methoden zur differenzierten entscheidungsorientierten Bewertung}

Inhaltlich gehört die entscheidungsorientierte Bewertung zur Präzisierung der „richtigen“ oder relevanten Kostenzuordnung (zu Entscheidungen). Es folgt somit dem Grundgedanken der Phase 3, bringt aber eine neue Komponente in Kalkulationen und entscheidungsorientierten Rechnungen ein: die Opportunitätskosten, und zwar in Form von verdrängten Deckungsbeiträgen. In der Literatur auch zu anderen betriebswirtschaftlichen oder insbesondere volkswirtschaftlichen Zusammenhängen zwar verschiedentlich schon vorher erwähnt, finden sich diese Größen erst ab den 1960er Jahren in konkreter fassbarer Weise und zwar durch die Verbreitung der Methoden des Operations Research (vgl. u. a. Hax 1965, Böhm/Wille 1966). Von manchem Kostenrechner eher zurückhaltend betrachtet, bringen sie Ergebnisse von Optimierungsrechnungen in die Kosten- und Leistungsrechnung ein. Hauptanwendungsfall sind planmäßig voll ausgelastete Produktionsanlagen. Will man jetzt entgegen dem bestehenden Produktionsplan andere Produkte auf diesen Maschinen herstellen, lohnt sich das finanziell nur, wenn die neu hinzutretenden Produkte pro Maschinenminute mindestens so viel Deckungsbeitrag einbringen, wie sie durch die wegfallenden Produkte verdrängen. Neben die manuell leichte Berechnung eines Deckungsbeitrags pro Engpasseinheit bei einer einzigen voll ausgelasteten Maschine treten lineare Planungsmodelle sowie diverse nichtlineare und ganzzahlige Ansätze. Sie erlauben es, die Opportunitätskosten präzise zu bestimmen, von denen vorher eher allgemein und nur prinzipiell die Rede war.

Aus den Ansätzen wird auch deutlich, dass die Berechnung situationsbezogen ist: Die Opportunitätskosten hängen als verdrängte Deckungsbeiträge davon ab, welche Aufträge konkret eingeplant waren. Fällt wegen Unterbeschäftigung und damit Nichtauslastung der vorhandenen Kapazitäten gar kein bisher eingeplanter Auftrag weg, bleiben die Opportunitätskosten bei null. Damit wird es möglich, von vielen Kostenrechnern bis heute kaum beachtet, die aus Phase 3 stammende Floskel von kurz- und langfristiger Preisuntergrenze einmal mehr zu präzisieren, und zwar durch die Preisuntergrenze bei freien und die bei ausgelasteten Kapazitäten. Eine Diskussion, wann die kurze Frist endet und die lange beginnt, erübrigt sich dadurch glücklicherweise gleichzeitig. Andererseits erwächst daraus eine bis heute noch bestehende Aufgabe, nämlich der Transfer dieser Zusammenhänge in alltagspraktikable Lösungen so, dass die in vielen Anwendungsfällen punktuell aufflammende unselige Vollkosten-Teilkosten-Diskussion schließlich auch hier als historische Zwischenphase und damit endgültig als erledigt betrachtet werden könnte.

\section{Ausdifferenzierung des Rechensystems auf Zwecke der Führung unter widrigen Umständen}

\subsection{Sechste Phase: Weiterentwicklung zu Kostenmanagement-Methoden}

Die sechste Phase kann man pauschal mit dem Schlagwort Kostenmanagement kennzeichnen. Wann sie genau beginnt, lässt sich im Rückblick kaum feststellen. Zugehörige Überle- 
gungen zu Einzelaspekten und ergänzende Hinweise zu methodischen Ansätzen finden sich schon seit Anbeginn der Kosten- und Leistungsrechnungsdiskussion. Spezielle und vor allem exklusive Ansätze indessen lassen sich vor allem in den 1970er und 1980er Jahren finden. Hier geht es zunächst explizit um die Kostenbudgetierung, später dann auch um das Target Costing, das Zielkostenmanagement. Beides hat gewichtige Vorläufer. So ist von Anbeginn an die Kostenstellenrechnung nicht nur als dokumentarische Nachrechnung, sondern auch als Planrechnung konzipiert. In Lehrbüchern unterscheidet man früh zwischen Prognose- und Vorgabe- (= Budget-) Kostenrechnung (so z. B. bei Kosiol 1964). Die teils extensiven Ausarbeitungen zur Abweichungsanalyse (vgl. hierzu führend vor allem Kloock 1988) belegen überzeugend, dass hier zumindest implizit von vorheriger Vorgabe und nachträglicher Kontrolle mit eben der zugehörigen Analyse ausgegangen wird.

Neu ist jetzt die Formulierung von Ansätzen, die speziell der Planung und Steuerung der Kosten dienen, also gerade nicht eine (möglichst genaue) Berechnung oder Prognose anstreben. In diese Kategorie sind beispielsweise das Zero-Base-Budgeting (Meyer-Piening 1980) und vor allem die Gemeinkostenwertanalyse (Roever 1980) für das stellenbezogene Kostenmanagement einzuordnen. Aber auch die Bedeutung der Prozesskostenrechnung (insbesondere in Verbindung mit dem Prozesskostenmanagement) darf nicht unterschätzt werden. Mit der Prozesskostenrechnung wird es eher als möglich angesehen, Kosten nicht nur kostenstellenorientiert, sondern speziell für wichtige Querschnittsfunktionen und -aufgaben zu planen und zu steuern. Ihr wird das Verdienst zugesprochen, grundsätzlich bekannte „strategische Kostentreiber“, wie Teilevielfalt, Lieferantenanzahl und allgemein Komplexität, stärker ins Bewusstsein gerückt zu haben.

Am markantesten wird der steuerungsorientierte Betrachtungswechsel indessen beim stückbezogenen Kostenmanagement sichtbar, und zwar durch das auch entsprechend klar bezeichnete Target Costing, das Zielkostenmanagement. Gemeinsames Merkmal der Ansätze zum Kostenmanagement ist die explizite Fundierung von Zielwerten, die sich dementsprechend auch nicht logisch „begründen“ oder durch Kausalketten „ableiten“ lassen. Hat man sie als Ausgangswerte aber akzeptiert, lassen sich für die Umsetzung der Zielerreichung durchaus kostenrechnerische Zusammenhänge heranziehen. Typisch dafür ist die „Umkehr" der Kalkulationsabfolge von Produkten im Target Costing.

\subsection{Siebte Phase: Genauere Differenzierung der Kosten- und Leistungsrechnung nach angestrebten Führungszielen}

Die sechste Phase kann in gewissem Sinn als Vorbereitung der siebten Phase angesehen werden. Hier wird genauer danach differenziert, an wen sich welche Kosteninformationen richten und welche Führungsziele damit erreicht werden sollen. Es geht also nicht mehr (nur) um ein undifferenziertes allgemeines Kostenmanagement („, was muss in welche Richtung getan werden? “). Vielmehr steht jetzt im Zentrum der Überlegung, an welche hierarchische Ebene des Betriebs, an welche Stelle und letztlich an welche Person die Informationen aus der Kosten- und Leistungsrechnung gerichtet sind. Diese Variante der Kostenrechnung könnte man als „verhaltens- oder performanceorientiert“ bezeichnen. Kosteninformationen dienen dabei der Koordination und Verhaltenssteuerung in Unternehmen. Dabei werden Entscheidungsträgern im Management, z. B. Bereichs- oder Abteilungsleitern, Kostenziele (in Form von Kennzahlen mit einer angestrebten Sollhöhe) vorgegeben, an denen sie später beurteilt (Performancemessung) und ggf. vergütet (Managemententlohnung) werden. 
Auch die Phase 7 hat, wie fast alle anderen, frühe Vorläuferansätze, die bis in die 1960er Jahre oder noch weiter zurückreichen. Zwar finden sich bereits bei Eugen Schmalenbach mit dem Begriff der pretialen Lenkung (Schmalenbach 1948) entsprechende Überlegungen, aber erst ab den 1980er Jahren und damit deutlich später etabliert sich mit dem Prinzipal-Agenten-Ansatz ein prominenter Theorierahmen zur Beschreibung der Verhaltenssteuerung mit Kosteninformationen. In die deutschsprachige Kostenrechnungsliteratur wird diese Sichtweise von Ewert, Wagenhofer und Pfaff eingeführt (vgl. z. B. Ewert 1992, Wagenhofer 1992, Pfaff 1993). Kosteninformationen (z. B. Kostenbudgets, Deckungsbeiträge, das operative Ergebnis, Rentabilitätskennzahlen oder Gewinnmargen) werden in diesem Bezugsrahmen als Performancemaße und die daran gekoppelten variablen Entlohnungsbestandteile als Teile eines Vertrages zwischen dem Prinzipal und dem Agenten interpretiert. Ziel ist es, Performancemaße derart zu gestalten und damit Entscheidungen dezentraler Verantwortungsbereiche derart zu steuern, dass die Gesamtunternehmensziele möglichst gut gefördert werden. In einem allgemeinen Sinn enthalten letztlich alle Systeme der Kostenrechnung Aspekte der Verhaltenssteuerung. Die Zurechnung von Verantwortlichkeiten für bestimmte Abweichungen, z. B. die Verantwortung der Kostenstellenleitung für die Verbrauchsabweichung (so bereits bei Kilger 1961), stellt nichts anderes als eine Verhaltenssteuerung durch Kosten dar, mit der die Kostenstellenleitung zu einem effizienten Ressourceneinsatz motiviert werden soll (vgl. Arbeitskreis Internes Rechnungswesen der Schmalenbach-Gesellschaft 2017).

So wird über den äußerlichen Berührpunkt hinaus auch eine inhaltliche Verbindung der Kosten- und Leistungsrechnung zum Controlling erreicht. Die Möglichkeiten der Kostenund Leistungsrechnung werden bei sinnvoller Anwendung damit stärker ihrer Rolle als betriebswirtschaftliches Führungsinstrument gerecht. Damit verbunden ist ein deutlicher sichtbares Unterscheiden von entscheidungsorientiertem und steuerndem gegenüber einem dokumentierendem Rechnungswesen.

Obwohl vielleicht nicht auf den ersten Blick sichtbar, gehören in diese Kategorie auch zwei „Dauerbrenner“ der Kosten- und Leistungsrechnung: die Kalkulation und die Periodenabrechnung. Die Kalkulation als stückbezogene Rechnung kann ja über alle Phasen der Kosten- und Leistungsrechnungs-Entwicklung hinweg als treuester Begleiter der jeweiligen Entwicklungsstufen angesehen werden. Sie hat dabei - regelmäßig weniger nach den methodischen Ansätzen als mehr durch die offensichtliche Interpretation belegt - häufiger die teils unbewusste Rolle geändert, aber eben nicht bei allen Beteiligten und auch nicht gleichzeitig. War die Kalkulation in den Anfängen der Kostenrechnung eine Verkaufspreisberechnung und damit Preisrechtfertigung (vgl. Pfaff/Weber 1998, S. 153), so ist sie, zweifellos damit erst präziser werdend, dann ab Phase $3 \mathrm{zu}$ einer Grenzkostenberechnung und später dann mit Phase 5 zu einer Preisuntergrenzen-Berechnung geworden.

In zahlreichen Fällen der betrieblichen Anwendung spielen die Kalkulationsergebnisse aber auch die Rolle einer Art Steuerungsvorgabe, insbesondere an den Verkauf. Es kann also gar nicht verwundern, dass auch noch im Jahr 2016 immer wieder leidenschaftliche Diskussionen entstehen, wenn Vertreter unterschiedlicher Zielsetzungen unbedarft aufeinandertreffen, vor allem ein preisuntergrenzen-orientierter Kostenrechner mit einem Manager, der Preisvorgaben an mengenorientierte Verkäufer setzt. Freilich haben die verschiedenen Phasen der sich entwickelnden und perfektionierenden Kosten- und Leistungsrechnung durchaus erhebliche Klarheit in die Kalkulationsdiskussion gebracht: Durch die Teilkostenrechnung (im Sinne einer Grenzkostenrechnung) ist es überhaupt erst möglich ge- 
worden, zielsetzungsfreie, „objektive“ Stückkosten zu berechnen, indem sie als zusätzlich anfallende Kosten (Grenzkosten) für eine zusätzliche Mengeneinheit definiert werden.

Nach den hier diskutierten steuernden Ansätzen spricht indessen nichts dagegen, für Steuerungszwecke, etwa Verkäufern gegenüber, einen Soll-Verkaufspreis vorzugeben. Freilich sollte der, wie alle Zielvorgaben, aus den Zielsetzungen, den vermuteten verhaltenssteuernden Wirkungen und zu vermeidenden Nebeneffekten her begründet und angesetzt werden, ggf. auch gegenüber alternativen Steuerungsvorgaben abgewogen werden. Keinesfalls lässt sich ein solcher steuernder Vorgabepreis aus Kostenrechnungskonzepten eindeutig „berechnen“, auch wenn das dem preisvorgebenden Management die Begründung der Vorgabenhöhe erleichtern oder ersparen würde. Die vielfach herangezogene Idee, hierzu eben „die“ Vollkosten zu nehmen, scheitert stets daran, dass es zwar - von Rechenfehlern und Rundungsfragen wegen unterschiedlicher Zurechnungspräzision abgesehen - eine eindeutige Grenzkosten-Preisuntergrenze gibt, aber eben nie eine eindeutige Vollkostenkalkulation. Bei jener werden ja bestimmte Mengenfixkosten willkürlich „zugeschlüsselt“, so dass mit dem veranschlagten Fixkostenbetrag und dessen Verteilungsschlüssel mindestens zwei Freiheitsgrade der Vollkostenkalkulation bestehen. Will man beauftragte Verkaufseinheiten steuern, kommen allerdings neben einer einfachen Preisvorgabe dafür auch andere Vorgabe-Größen in Frage, etwa kombinierte Mengen-Umsatzvorgaben oder eine Deckungsbeitrags-Summen-Vorgabe, die dann ggf. mit anderen Vorgabegrößen verbunden werden, ohne dass die Gesamtvorgabe dadurch gleich kompliziert oder unübersichtlich würde.

Der zweite Fall betrifft die interne Periodenabrechnung. Steuerungsziel ist z. B. die Vorgabe einer Mindest-Zielhöhe, regelmäßig verbunden mit einer damit korrespondierenden Perioden-Bonuszahlung. Auch diese Idee ist weder neu, noch haben sich überraschende Verfahrensweisen dazu entwickelt. Sie ist aber unter der Bezeichnung „wertorientierte Unternehmenssteuerung “ insbesondere ab Mitte der 1990er Jahre mit großen Präzisierungsbemühungen in den Mittelpunkt der Diskussion um die Manager- und Konzernsteuerung gerückt. Wie fast immer kommt der Anstoß dazu aus den Usancen und Notwendigkeiten (vor allem Engpässen) der Praxis. Im Fall der wertorientierten Steuerung ist es der bis dahin in der Praxis übliche Fokus auf das Betriebsergebnis oder den Reingewinn bei gleichzeitiger Ignoranz des in einem Unternehmen gebundenen Kapitals - obwohl schon im 19. Jahrhundert nicht nur Kostenrechnern klar ist, dass gebundenes Kapital Kosten verursacht (kalkulatorische Zinsen). Erst als (Eigen-)Kapital in den 1990er Jahren aufgrund einer boomenden M\&A-Tätigkeit zum bedeutendsten Engpass wird, ändert sich der Fokus. Populär werden Residualgewinne wie der Economic Value Added (EVA), der Economic Profit oder der Cash Value Added (CVA), die zeigen sollen, ob es Unternehmen gelingt, nicht nur Gewinn zu erzielen, sondern auch die „Kapitalkosten“ zu erwirtschaften. Zudem sollen Werttreiber (Value Driver) identifiziert und gesteuert werden, um das Unternehmen am Kapitalmarkt attraktiver zu machen.

Aber auch die wertorientierte Steuerung muss sich mit einem der Hauptprobleme periodenbezogener Rechnungen auseinandersetzen, nämlich der (Zwischen-)Abrechnung eines Jahres, wo doch regelmäßig überjährige Projekte dies prinzipiell unmöglich machen („,wie kann man denn abschließen, solange man mitten im Leben steht" - Rieger 1928, S. 209). $\mathrm{Zu}$ dieser Aufgabe liefert die oft planerisch und entscheidungsorientiert ausgerichtete Kosten- und Leistungsrechnung, wenn überhaupt, nur teilweise Antworten. Und das verbindet sie an dieser Stelle mit der externen Rechnungslegung, das „entscheidungsnützliche“ Infor- 
mationen für die Jahresabschlussadressaten bereitstellen soll. Wiederum bereichert der Blickwinkel der Verhaltenssteuerung die Diskussion, wird doch erneut deutlich gemacht, dass es hier eben nicht um eine zweckneutrale, möglichst objektive Abbildung der wirtschaftlichen „Realität“ gehen kann. Vielmehr wird die Frage gestellt, wie dezentrale Einheiten in einem Unternehmen mit Hilfe wertorientierter Periodenergebnisse zu führen sind, damit sie Investitionen mit positivem Kapitalwert tätigen. Diese nicht unwichtige Problematik ist vor allem dann von Bedeutung, wenn der Zeithorizont des Managements sich von dem der Investoren unterscheidet, in der Regel kurzfristiger ausfällt („Problem des ungeduldigen Managers"). Die passende Berücksichtigung von Zinsen und die Einhaltung des pagatorischen oder Kongruenzprinzips stellen dann sicher, dass der Barwert der Residualgewinne eines Investitionsprojekts dessen Kapitalwert entspricht (Conservation Property; vgl. Preinreich 1938; Lücke 1955). Zudem geht es darum, die Methoden des Rechnungswesens (zum Beispiel Abschreibungsverfahren, Vorratsbewertung, Bilanzierung bei langfristiger Auftragsfertigung) so festzulegen, dass kapitalwertsteigernde Investitionen die (Messung der) Residualgewinne in jeder Periode erhöhen (Rogerson 1997, 2008; Reichelstein 2000).

Die wertorientierte Unternehmensführung befeuert auch die wohl letztlich von Ziegler 1994 ausgelöste und seitdem schwelende Diskussion um eine Harmonisierung des Rechnungswesens (siehe hierzu auch Kapitel 5 unten). Da alle anderen Rechenzwecke und Betrachtungsrichtungen inhaltlich zu weit auseinanderliegen, ist damit insbesondere (und zwangsläufig nur) eine Angleichung externer und interner Regeln für die Perioden- und Projektabrechnung gemeint (vgl. auch Pfaff 1994).

\section{Zukunftsaspekte des internen Rechnungswesens}

\subsection{Was bleiben wird}

Fragt man Praktiker nach den zentralen Fragestellungen, auf die das interne Rechnungswesen antworten muss, erhält man vor allem folgende Antworten (Arbeitskreis Internes Rechnungswesen der Schmalenbach-Gesellschaft 2017):

- Was kostet mein Produkt?

- Was verdiene ich?

- Wie setze ich meine Ressourcen ein?

- Wie kann ich meine Kosten beeinflussen (managen)?

- Wie steuere ich mit Kosteninformationen das Verhalten meiner Mitarbeiter?

Der Leser dieses Beitrags stellt fest, dass es genau diese Fragen sind, welche die Entwicklung der Kosten- und Leistungsrechnung maßgeblich getrieben haben. Es sind Fragen, die mit dem Erfolg des betrieblichen Handelns zu tun haben und immer und zu jeder Zeit von Bedeutung sind. Deshalb hängt die Kostenrechnung nicht von Modeströmungen ab. Auf dieser Grundlage wagen wir die Prognose, dass sich an den zentralen Fragestellungen der Kosten- und Leistungsrechnung auch in den nächsten (70) Jahren wenig ändern wird, unabhängig davon, wie sich die Geschäftsmodelle der Unternehmen, ihre Engpässe oder die Interessen ihrer Stakeholder verändern mögen.

Wahrscheinlich erhält aber die eine oder andere Fragestellung eine neue oder veränderte Dimension. So könnten die Fragen nach den Kosten eines Produkts, aber auch nach dem Erfolg eines Unternehmens oder Bereichs sowie nach dem Ressourceneinsatz zunehmend 
durch die Dimensionen Ökologie und Soziales geprägt sein, was sich heute unter dem Schlagwort der Nachhaltigkeitsberichterstattung und -steuerung andeutet. Noch stehen wir ganz am Anfang dieser Entwicklung. Auch das Kostenmanagement und die dezentrale Steuerung mit Kosten- und Leistungsinformationen werden Dauerbrenner der Diskussion bleiben. Gleichzeitig ist jedoch zu vermuten, dass sich wandelnde Geschäftsmodelle und Rahmenbedingungen wie die digitale Revolution, in der wir uns gegenwärtig befinden, zu einer Weiterentwicklung der methodischen Kostenrechnungskompetenz führen werden.

\subsection{Methodische Weiterentwicklungen im Hinblick auf betriebliche Angebote des} 21. Jahrhunderts

Gegenwärtig sehen wir vor allem folgende Hauptentwicklungen:

(1) Weiterentwicklung der Kosten- und Leistungsrechnung innerhalb eines einheitlichen internen Rechnungswesens;

(2) Differenziertere Abbildung gerade von Größen mit besonders sichtbar entscheidungsrelevanter Bedeutung;

(3) Rechnerische Behandlung von Angeboten, die in das herkömmliche Schema des punktuellen Sachgutverkaufs nicht passen;

(4) Erweiterung der Kostenrechnung um ökologische und soziale Dimensionen;

(5) Fortschreitende Digitalisierung der Kostenrechnung und des Rechnungswesens allgemein;

(6) Weitere Ausgestaltung des gesamten internen Rechnungswesens als Controlling-Instrument.

$\mathrm{Zu}$ (1): Weiterentwicklung der Kosten- und Leistungsrechnung innerhalb eines einheitlichen internen Rechnungswesens

Schon seit einigen Jahren zeichnet sich ein starkes Zusammenwachsen der verschiedenen Teile des internen Rechnungswesens zu einem integrierten Gesamten ab. In besonderem Maße gilt dies für den Zusammenhang von Kosten- und Leistungsrechnung einerseits und Investitionsrechnung andererseits. Dafür gibt es mehrere Argumente (vgl. vor allem Troßmann 2013). Einer der auffälligsten Gründe dafür liegt in der immer feineren Ausgestaltung der entscheidungsorientierten Kosten- und Leistungsrechnung als Teilkostenrechnung. Dadurch werden die Sunk Costs bereits früher getroffener Entscheidungen in die laufende Rechnung korrekterweise nicht mehr einbezogen. Was etwa für die stückbezogene Kalkulation und auch für die periodenbezogene Auflagenentscheidung richtig ist, wirft aber umso mehr die Frage auf, an welcher Stelle man sich um diese jetzt nicht mehr disponiblen Kosten eigentlich hätte kümmern sollen. Das begründet eine präziser fundierte und detaillierter ausgestaltete Investitionsrechnung. Bei konsequenter Fortführung dieses Arguments ist es geradezu zwangsläufig, weitere periodenübergreifende Projektentscheidungen unter einem investitionsrechnerischen Blickwinkel zu betrachten, also Entscheidungen über die Produktentwicklung generell, über Konstruktionsdetails, über den Markteintritt, über periodenweise Bereitstellung der Produktionskapazität usw. Solche übergreifenden, mehrperiodigen Rechnungen zu den grundlegenden Entscheidungen ersetzen mehr und mehr pauschale Fixkostenpositionen in nachgeordneten Rechnungen, soweit jene dort nicht mehr disponibel sind. 
$\mathrm{Zu}$ (2): Differenziertere Abbildung gerade von Größen mit besonders sichtbar entscheidungsrelevanter Bedeutung

Der zweite Punkt betrifft einzelne Entscheidungsgrößen, die aber von großer relativer Entscheidungsrelevanz sind, wenn man ihre rechnerischen Auswirkungen betrachtet. Dazu gehören insbesondere Positionen, die in der Vergangenheit und auch bis heute eher stiefmütterlich und insgesamt vereinfachend behandelt wurden. Typische Beispiele liegen dort, wo die vermeintlichen oder nach der bisherigen Erfahrung die tatsächlichen Größenordnungen in eher unauffälligem Rahmen lagen. Drei Gruppen solcher Fälle lassen sich unterscheiden:

(a) Kosten, die für zufällige und nicht beeinflussbare Ereignisse anfallen, etwa sogenannte Wagniskosten, Forderungsausfälle, vorzeitiger Maschinenverschleiß: Soweit nicht ohnehin die Risikofolgen extern versichert werden, kommt hier, wenn auch sicher noch zögerlich, die Übertragung von Versicherungskalkulationen auf derartige Fälle in Frage. Ein spezieller Ansatz, der auf einer ganz anderen Überlegung beruht, kann an dieser Stelle ebenfalls aufgegriffen und ggf. nach passender Spezialisierung hier angewendet werden: die Idee der investitionsrechnerisch begründeten Kostenberechnung, wie sie vor allem Küpper (1985) schon Mitte der 1980er Jahre vorgeschlagen hat, der aber bisher kaum breiter aufgegriffen wurde. Das Konzept ist bisher vor allem für Maschinenkosten ausformuliert, aber von hinreichender Allgemeinheit für eine prinzipiell breitere Anwendung.

(b) Kosten und Leistungen, die für zufällige „kundenbezogene“ Ereignisse anfallen: Dies sind vor allem nachlaufende Kosten und Erlöse in mehrperiodigen Kundenbeziehungen, etwa spätere Gewährleistungskosten oder Deckungsbeiträge aus dem Nachverkauf von Verbrauchsmaterial. Solche Positionen werden mit Verweilzeitfaktoren erfasst - einem bislang in kostenrechnerischen Zusammenhängen unüblichen Begriff, mit dem sich die vermuteten Auftretenshäufigkeiten nachlaufender Positionen adäquat abbilden lassen. Die so prognostizierten Nachlaufkosten und -erlöse gehen in die unter Punkt 3 besprochenen umfassenderen Produktlebenszyklusrechnungen ein.

(c) Zinskosten sowie Wirkungen, die aus der Zinsberïcksichtigung in mehrperiodigen Rechnungen resultieren: Die hohe Sensibilität, die durchweg Ergebnisse mehrperiodiger Rechnungen, vor allem Kapitalwerte, auf die Zinshöhe zeigen, legt es nahe, dem Kalkulationszinssatz in internen Rechnungen generell eine stärkere Bedeutung beizumessen. Die früher übliche Festlegung eines betrieblichen Kalkulationszinssatzes, der dann oft über Jahre hinweg in gleicher Höhe (für alles) verwendet wurde, könnte deshalb einer differenzierteren Betrachtung weichen. Innerhalb der Investitionsrechnung haben sich in jüngster Zeit verschiedene Ansätze herausgebildet, die eine adäquate Berücksichtigung der betrieblichen Finanzierungssituation in entscheidungsorientierten Rechnungen erlauben. Beispielsweise kann der im externen Rechnungswesen insbesondere bei der Unternehmensbewertung übliche gewichtete Mischzinssatz (WACC) im regelmäßig besser fundierbaren internen Rechnungswesen durch eine intervallweise Verwendung exakter tatsächlicher Zinssätze ersetzt werden. Aus den in der konkreten Entscheidungssituation vorliegenden Finanzalternativen lassen sich ohne besondere rechnerische Umständlichkeiten projektbezogene periodenindividuelle Zinssätze errechnen, die zu einer situationsadäquaten und laufzeitentsprechenden Zinsberücksichtigung herangezogen werden können (vgl. Troßmann 2013). Beispielrechnungen zei- 
gen mühelos, dass selbst und gerade eine langdauernde Niedrigzinsphase gute Argumente gegen einen allzu pauschalen betrieblichen Kalkulationszinssatz liefert.

$\mathrm{Zu}$ (3): Rechnerische Behandlung von Angeboten, die in das herkömmliche Schema des punktuellen Sachgutverkaufs nicht passen

Vielfach steht hinter den klassischen Ansätzen der Kosten- und Leistungsrechnung das Bild eines Sachguts aus industrieller Fertigung. Unbewusst messen Kostenrechner bis heute die Leistungsfähigkeit methodischer Ansätze an den daraus resultierenden Anforderungen. Unterdessen ist die Angebotswelt erheblich vielfältiger geworden. In mehreren Fällen ist man sich auch erst in jüngerer Zeit einer solchen vielleicht schon länger bestehenden Vielfalt bewusst geworden. Letzteres gilt auf jeden Fall für Lebenszyklusrechnungen, mit denen Produkte rechnerisch abgebildet werden, die zum Verkaufszeitpunkt nur einen Teil der tatsächlich mit dem Verkauf verbundenen Zahlungsströme auch schon realisiert haben. Vielfältige Nachlaufkosten und -erlöse erfordern einen umfassenden mehrperiodigen Ansatz zur Komplettbeurteilung derartiger Größen. Dies integriert die bereits unter Punkt 2 genannten Positionen in einem umfassenden Ansatz. Die Deckungsbeitragsrechnung mit ihrer Unterscheidung von stückbezogenem Deckungsbeitrag für stückbezogene Entscheidungen und ggf. mehrstufig differenzierten Gesamtdeckungsbeiträgen für stellenbezogene Bereitstellungsentscheidungen wird mit dem Ansatz der Lebenszyklusrechnung auf einen mehrperiodigen Entscheidungszusammenhang erweitert. So entstehen Stückdeckungsbeitrags-Barwerte einerseits und Gesamtdeckungsbeitrags-Kapitalwerte andererseits. Je mehr betriebliche Angebote eine mehrperiodige Kundenbindung anstreben, desto wichtiger werden derartige Rechnungen in der Zukunft. Die vorliegenden Grundansätze zur Lebenszyklusrechnung werden möglicherweise deshalb nicht nur mehr Anwendungsfelder finden, sondern sich auch weiter ausdifferenzieren.

Zwei andere, sehr unterschiedliche Aspekte lassen ebenfalls deutliche Erweiterungen des herkömmlichen typischen Ansatzes der Kosten- und Leistungsrechnung erwarten: Einmal sind es die generellen Nachhaltigkeitsbestrebungen, zum anderen die Veränderung der betrieblichen Angebote selbst.

Der Nachhaltigkeitsgedanke hat im hier betrachteten Zusammenhang zunächst innerbetriebliche Wirkungen: Bisher als Abfall oder Abwärme entstandene Nebenwirkungen einer Produktion werden mehr und mehr als Kuppelprodukte aufgefasst, weil sie weiterverarbeitet werden und dann ggf. in veränderter Form als Absatzgüter auftreten. Damit rücken die Ansätze der Kuppelproduktion aus Phase 4 wieder näher in das aktuelle Blickfeld. Vermutlich müssen sie aufgefrischt und ggf. an die heutige Situation angepasst werden.

Das allgemein veränderte Produktangebot zielt künftig mehr als bisher auf die Problemlösung des Kunden ab, weniger dagegen auf den Verkauf eines bestimmten Einzelprodukts. Beispielsweise wird der Verkauf eines Autos ersetzt durch die Sicherstellung einer bestimmten Transportleistung mit definiertem Komfort. Was also angeboten wird und demgemäß auch kalkuliert werden muss, ist eine Verbundleistung, ein Bündel ganz unterschiedlicher Teilleistungen, etwa ein Auto, die Eventualbereitstellung eines Ersatzautos, die Wartung, Reparatur, die Verfügbarkeit von Internetkommunikationsleistungen während der Reise sowie ggf. Autoschutzbriefleistungen. Dies hat auch Auswirkungen auf die Erlösseite. So fordert IFRS 15 (für die externe Rechnungslegung, aber mit Konsequenzen für das interne Rechnungswesen), eigenständig abgrenzbare Leistungsverpflichtungen eines Vertrags zu identifizieren und den Transaktionspreis auf die einzelnen Leistungsverpflichtungen zu verteilen. Ganz abgesehen davon, dass der Transaktionspreis mit all seinen 
Komponenten (variable Gegenleistungen, Finanzierungskomponente usw.) gar nicht einfach bestimmbar ist, stellt seine Aufteilung auf die einzelnen Leistungsverpflichtungen eine weitere Hürde dar, bei deren Überwindung die Kosten- und Leistungsrechnung mit ihrer Methodenkompetenz, etwa aus der Kuppelkalkulation, wertvolle Hilfestellung leisten kann.

\section{$\mathrm{Zu}$ (4): Erweiterung der Kostenrechnung um ökologische und soziale Dimensionen}

Das Bekenntnis zur Nachhaltigkeit ist gegenwärtig wohl eher noch ein Schlagwort oder Wunschdenken denn gelebter Alltag. Und dennoch gibt es bereits Beispiele, wie ein „Green Controlling " in der Kosten- und Leistungsrechnung aussehen könnte (vgl. Zimmermann 2015). Das dort beschriebene Unternehmen nutzt verbrauchs- und erzeugungsrelevante Daten der Stromversorgung in Echtzeit, um die Maschinenbelegung zu verbessern, Spitzenlasten im Produktionsablauf zu vermeiden und $\mathrm{CO}_{2}$-Emissionen zu reduzieren. Ziel ist es zudem, Energieeffizienz direkt in den Kalkulationsprozess zu integrieren und auftragsbezogene Energiesparerfolge oder -verluste transparent zu machen.

$\mathrm{Zu}$ (5): Fortschreitende Digitalisierung der Kostenrechnung und des Rechnungswesens allgemein

Die zunehmende Digitalisierung von Prozessen führt zu einem (internen) Rechnungswesen, das Buchen, Planen, Analysieren und Steuern in Echtzeit erlaubt. Das eben angeführte Beispiel eines Green Controlling zeigt bereits die (unmittelbare) Verknüpfung von realwirtschaftlichen Vorgängen und Daten mit der Kostenarten-, -stellen- und -trägerrechnung. Was diese Möglichkeiten für die Entscheidungsfindung und -steuerung bedeutet, kann momentan nur erahnt werden. Sicher ist aber, dass es beispielsweise sehr viel einfacher sein wird, situationsabhängige Opportunitätskosten in der Kostenrechnung abzubilden und dies gewinnbringend zu nutzen. Geschäftschancen und -risiken können zeitnäher und zuverlässiger beurteilt und berücksichtigt werden. Rechnungswesen in Echtzeit heißt zudem, dass Ist- und aktualisierte Prognosewerte aller relevanten Bezugsobjekte jederzeit abgerufen und beurteilt werden können. Das Erkennen von Abweichungen und ein entsprechendes Gegensteuern werden damit deutlich einfacher, stellen andererseits aber auch erhöhte Anforderungen an die Auswahl und Definitionspräzision relevanter Schlüsselindikatoren. Digitalisierung könnte zudem auch bedeuten, dass es für das Management bedeutend schwieriger wird, Performancemaße zu manipulieren. Welche Rolle dabei die neue „Blockchain"-Technologie spielen wird, bleibt abzuwarten (Yermack 2015).

$\mathrm{Zu}$ (6): Weitere Ausgestaltung des gesamten internen Rechnungswesens als ControllingInstrument

Der letzte Ansatzpunkt schließlich richtet sich auf die Bedeutung des internen Rechnungswesens als Controlling-Instrument generell. Hier zeichnet sich in langfristiger Entwicklung immer stärker eine unterstützende Rolle ab, die dem internen Rechnungswesen für das Controlling zukommt. Immer mehr wird es zum betrieblichen Standard, dass Ergebnisse des internen Rechnungswesens vor allem über und durch den Controller an das Management herangetragen werden. Damit tritt der instrumentelle Charakter des internen Rechnungswesens stärker hervor. Wie sich das auf die relative Bedeutung einzelner methodischer Komponenten des internen Rechnungswesens auswirkt, muss sich zeigen. Es hängt davon ab, welche Art der Führungskoordination im Betrieb besteht, wie geführt wird, auf welcher Basis Vorgaben formuliert, Kontrollen angesetzt und Manager honoriert werden. Das wird die Rolle des internen Rechnungswesens und damit ihres ursprünglichen Kerngebiets, der Kostenrechnung, deutlich bestimmen. 


\subsection{Neue Geschäftsmodelle - Anforderungen an Organisation, Rechnungswesen und Verhaltenssteuerung}

Schon in den Überlegungen des Abschnitts 5.2 zeigt sich, dass die Angebotswelt der Unternehmen sehr viel komplexer geworden ist. Der Verkauf einfacher Sachgüter wird abgelöst durch das Angebot ganzer Leistungsbündel und vielfältiger Vertragsverpflichtungen. Diese sind aber häufig nicht auf einen Produkt- oder Geschäftsbereich eines Unternehmens beschränkt, sondern umfassen Sach- und Dienstleistungen mehrerer Geschäftsbereiche und bündeln sie (neu) für den Markt. Die Einführung solcher geschäftsbereichsübergreifender strategischer Geschäftsfelder erzeugt Anpassungsdruck auf die Organisation eines Unternehmens und dessen (internes) Rechnungswesen. Damit die Entwicklung und Förderung neuer Arten von Geschäftsmodellen bestmöglich im Unternehmen vorangetrieben werden können, müssen Organisation, Rechnungswesen und Incentivierung eng aufeinander abgestimmt sein. Dass dafür insbesondere auch die Konzepte der Kosten- und Leistungsrechnung eine treibende Kraft entwickeln können, zeigen beispielsweise eindrücklich die betrieblichen Umsetzungen der Kostenstellenbildung als Verantwortungseinheiten in den 1960er Jahren und die Konsequenzen der Prozesskostenrechnungsdiskussion in den 1990er Jahren.

Sollen Mitarbeiter motiviert werden, ihre Aufmerksamkeit verstärkt auf geschäftsbereichsübergreifende Aktivitäten zu legen, müssen organisatorisch Entscheidungskompetenzen neu geordnet werden. Gleichzeit besteht eine enge Verzahnung zwischen der Zuordnung von Entscheidungsrechten (als Frage der Organisation) und der Messung, wie erfolgreich diese Rechte ausgenutzt wurden (Definition von Performancemaßen als Frage des internen Rechnungswesens). Die Zurechnung von Kosten und Erlösen ist dabei ein zentraler Aspekt innovativer Geschäftsmodelle; sie wird über ihren Erfolg maßgeblich mitentscheiden. Dass sie so eine Rolle übernimmt, wäre nicht das erste Mal in der langen Geschichte der Kosten- und Leistungsrechnung.

\section{Literaturverzeichnis}

Adam, Dietrich (1970): Entscheidungsorientierte Kostenbewertung, Wiesbaden.

Agthe, Klaus (1959): Stufenweise Fixkostendeckung im System des Direct Costing, in: Zeitschrift für Betriebswirtschaft, Jg. 29, Nr. 3, S. 404-418.

Arbeitskreis Internes Rechnungswesen der Schmalenbach-Gesellschaft (Hrsg.) (2017): Säulen der Kostenrechnung - Originalquellen und Kommentierungen zu den Grundfragen und Konstruktionsprinzipien der Kostenrechnung, München.

Ballewski, Albert (1880): Die Calculation für Maschinenfabriken, 2. Aufl., Magdeburg.

Böhm, Hans-Hermann/Wille Friedrich (1966): Deckungsbeitragsrechnung, Grenzpreisrechnung und Optimierung, 6. Aufl., München 1977 (Erstauflage 1966).

Bohr, Kurt (1988) Zum Verhältnis von klassischer Investitions- und Erlösrechnung, in: Zeitschrift für Betriebswirtschaft, Jg. 58, S. 1171-1180.

Clark, John Maurice (1923): Studies in the Economics of Overhead Costs, Chicago.

Coase, Ronald Harry (1938): Business Organization and the Accountant, New York.

Cooper, Robin (1989): The Rise of Activity-Based Costing - Part Four: What do Activity-Based Costs Systems Look Like? in: Journal of Cost Management, Jg. 3, Nr. 1, S. 38-49. 
Cooper, Robin/Kaplan, Robert S. (1988): Measure Costs Right: Make the Right Decisions, in: Harvard Business Review, Jg. 66, S. 96-103.

Demski, Joel S./Feltham, Gerald A. (1976): Cost Determination: A conceptual approach, Ames.

Dorn, Gerhard (1961): Die Entwicklung der industriellen Kostenrechnung in Deutschland, Berlin.

Drumm, Hans-Jürgen (1972): Theorie und Praxis der Lenkung durch Preise, in: Zeitschrift für betriebswirtschaftliche Forschung, Jg. 24, S. 253-267.

Ewert, Ralf (1992): Controlling, Interessenkonflikte und asymmetrische Information, in: Betriebswirtschaftliche Forschung und Praxis, Jg. 44, S. 277-303.

Franz, Klaus-Peter (1990): Die Prozesskostenrechnung - Darstellung und Vergleich mit der Plankosten- und Deckungsbeitragsrechnung, in: Ablert, Dieter/Franz, Klaus-Peter/Göppl, Hermann (Hrsg.): Finanz- und Rechnungswesen als Führungsinstrument, Wiesbaden, S. 109-136.

Fredersdorff, Leopold Friedrich (1802): Praktische Anleitung zu einer guten Eisenhüttenökonomie, Pyrmont.

Friedl, Birgit (2004): Kostenrechnung - Grundlagen, Teilrechnungen und Systeme, 2. Aufl., München, Wien 2010 (Erstauflage 2004).

Harris, Jonathan (1936): What did we earn last month? in: N. A. C. A. Bulletin, Jg. 17, S. 501-527.

Hax, Herbert (1965): Kostenbewertung mit Hilfe der mathematischen Programmierung, in: Zeitschrift für Betriebswirtschaft, Jg. 35, S. 197-210.

Heinen, Edmund (1956): Die Kosten, Saarbrücken.

Hiromoto, Toshiro (1988): Another Hidden Edge - Japanese Management Accounting, in: Harvard Business Review, Jg. 66, S. 22-26.

Hopwood, Anthony G. (1974): Accounting and Human Behavior, London.

Horngren, Charles T. (1962): Cost Accounting - A Managerial Emphasis, 15. Aufl., Saddle River (NJ) 2015 (Erstauflage 1962).

Horváth, Peter/Mayer, Reinhold (1989): Prozesskostenrechnung - Der neue Weg zu mehr Kostentransparenz und wirkungsvolleren Unternehmensstrategien, in: Controlling, Jg. 1, S. 214-219.

Hummel, Siegfried/Männel, Wolfgang (1978): Kostenrechnung Band 1 und Band 2: Grundlagen, Aufbau und Anwendung, 4. Aufl., Wiesbaden 1993 (Erstauflage 1978).

Illetschko, Leopold L. (1941): Praktische Kostenrechnung, Wien.

Jacob, Herbert (1971): Zur optimalen Planung des Produktionsprogramms bei Einzelfertigung, in: Zeitschrift für Betriebswirtschaft, Jg. 41, S. 495-516.

Jung, Johann Heinrich (1786): Anleitung zur Cameral-Rechnungswissenschaft, Leipzig.

Käfer, Karl (1955): Standardkostenrechnung, Zürich.

Kilger, Wolfgang (1961): Flexible Plankostenrechnung und Deckungsbeitragsrechnung, Wiesbaden (inzwischen mit weiteren Autoren in der 13. Auflage, 2012, erschienen).

Kloock, Josef (1988): Erfolgskontrolle mit der differenziert-kumulativen Abweichungsanalyse, in: Zeitschrift für Betriebswirtschaft, Jg. 58, S. 423-434.

Koch, Helmut (1948): Die industrielle Kostenkalkulation als Stufenrechnung: ein Beitrag zur Theorie der Kalkulationsformen, Diss. Hannover.

Kosiol, Erich (1927): Kostenauflösung und proportionaler Satz, in: Zeitschrift für handelswissenschaftliche Forschung, Jg. 21, S. 345-358.

Kosiol, Erich (1951): Verrechnung innerbetrieblicher Leistungen, Wiesbaden.

Kosiol, Erich (1964): Kostenrechnung, Wiesbaden.

Kruschwitz, Lutz (1973): Die Kalkulation von Kuppelprodukten, in: krp, Jg. 17, S. 219-230. 
Küpper, Hans-Ulrich (1985): Investitionstheoretische Fundierung der Kostenrechnung, in: Zeitschrift für betriebswirtschaftliche Forschung, Jg. 37, S. 26-46.

Laßmann, Gert (1968): Die Kosten- und Erlösrechnung als Instrument der Planung und Kontrolle von Industriebetrieben, Düsseldorf.

Lehmann, Max Rudolf (1950): Die Problematik der Preispolitik auf Grenzkosten- und auf Vollkostenbasis, in: Zeitschrift für Betriebswirtschaft, Jg. 20, S. 332-338.

Leitner, Friedrich (1913): Die Selbstkostenberechnung industrieller Betriebe, 4. Aufl., Frankfurt a.M.

Lücke, Wolfgang (1955): Investitionsrechnungen auf der Grundlage von Ausgaben oder Kosten? in: Zeitschrift für handelswissenschaftliche Forschung, Jg. 7, S. 310-324.

Mahlert, Arno (1976): Die Abschreibungen in der entscheidungsorientierten Kostenrechnung, Diss. Saarbrücken.

Messerschmitt, Alfred (1884): Die Calculation der Eisenconstructionen, Essen.

Meyer-Piening, Arnulf (1980): Gemeinkosten senken - aber wie? in: Zeitschrift für Betriebswirtschaft, Jg. 50, S. 691-698.

Nicklisch, Heinrich (1922): Wirtschaftliche Betriebslehre, Stuttgart.

Norden, Helmut (1941): Der Betriebsabrechnungsbogen, Berlin, Stuttgart.

Peiser, Herbert (1923): Grundlagen der Betriebsrechnung in Maschinenbauanstalten, 2. Aufl., Berlin.

Pfaff, Dieter (1993): Kostenrechnung, Unsicherheit und Organisation, Heidelberg.

Pfaff, Dieter (1994): Zur Notwendigkeit einer eigenständigen Kostenrechnung - Anmerkungen zur

Neuorientierung des internen Rechnungswesens im Hause Siemens, in: Zeitschrift für betriebswirtschaftliche Forschung, Jg. 46, S. 1065-1084.

Pfaff, Dieter/Weber, Jürgen (1998): Zweck der Kostenrechnung? Eine neue Sicht auf ein altes Problem, in: Die Betriebswirtschaft, Jg. 58, S. 151-165.

Plaut, Hans-Georg (1953): Die Grenz-Plankostenrechnung, in: Zeitschrift für Betriebswirtschaft, Jg. 23, S. 347-363.

Preinreich, Gabriel (1938): Annual Study of Economic Theory: The Theory of Depreciation, in: Econometrica, Jg. 6, S. 219-241.

Reichelstein, Stefan (2000): Providing Managerial Incentives: Cash Flows versus Accrual Accounting, in: Journal of Accounting Research, Vol. 38, No. 2, S. 243-269.

Riebel, Paul (1956): Die Gestaltung der Kostenrechnung für Zwecke der Betriebskontrolle und Betriebsdisposition, in: Zeitschrift für Betriebswirtschaft, Jg. 26, S. 278-289.

Riebel, Paul (1964): Einzelkosten- und Deckungsbeitragsrechnung, Wiesbaden 1964.

Rieger, Wilhelm (1928): Einführung in die Privatwirtschaftslehre, Nürnberg.

Roever, Michael (1980): Gemeinkosten-Wertanalyse - Erfolgreiche Antwort auf die GemeinkostenProblematik, in: Zeitschrift für Betriebswirtschaft, Jg. 50, S. 686-690.

Rogerson, William P. (1997): Intertemporal Cost Allocation and Managerial Investment Incentives: A Theory Explaining the Use of Economic Value Added as a Performance Measure, in: Journal of Political Economy, Vol. 105, No. 4, S. 770-795.

Rogerson, William P. (2008): Intertemporal Cost Allocation and Investment Decisions, in: Journal of Political Economy, Vol. 116, No. 5, S. 931-950.

Rummel, Kurt (1934): Grundlagen der Selbstkostenrechnung, Düsseldorf.

Schär, Johann Friedrich (1911): Allgemeine Handelsbetriebslehre, Leipzig.

Schmalenbach, Eugen (1899): Buchführung und Kalkulation im Fabrikgeschäft - Unveränderter Nachdruck aus der Deutschen Metallindustrie-Zeitung von 1899, Jg. 15, Leipzig. 
Schmalenbach, Eugen (1919): Selbstkostenrechnung, in: Zeitschrift für handelswissenschaftliche Forschung, Jg. 13, S. 257-299.

Schmalenbach, Eugen (1948): Pretiale Wirtschaftslenkung - Band 2: Pretiale Lenkung des Betriebes, Bremen-Horn.

Schmidt, Fritz (1923): Der Wiederbeschaffungspreis des Umsatztages in Kalkulation und Volkswirtschaft, Berlin.

Schneider, Erich (1941): Die innerbetriebliche Leistungsverrechnung in der Kostenarten- und Kostenstellenrechnung, Zeitschrift für handelswissenschaftliche Forschung, Jg. 35, S. 251-275.

Schnettler, Albert (1932): Die Rechnung mit Äquivalenzziffern in der Praxis, in: Zeitschrift für handelswissenschaftliche Forschung, Jg. 26, S. 310-326.

Schweitzer, Marcell/Küpper Hans-Ulrich (1974): Systeme der Kosten- und Leistungsrechnung, 1. Aufl., München (inzwischen mit weiteren Autoren in der 11. Aufl., 2016, erschienen).

Seidenschwarz, Werner (1993): Target Costing - Marktorientiertes Zielkostenmanagement, München.

Tanaka, Masayasu (1989): Cost Planning and Control Systems in the Design Phase of a New Product, in: Monden, Yasubiro/Sakurai, Michiharu (Hrsg. 1989): Japanese Management Accounting, Cambridge, Norwalk, S. 49-71.

Tolkmitt, H. (1894): Grundriß der Fabrik-Geschäftsführung - Ein Leitfaden zum praktischen Gebrauch für Fabrikbesitzer und Angestellte sowie zum Selbstunterricht für junge Kaufleute und Techniker, Leipzig.

Troßmann, Ernst (1992): Flexible Plankostenrechnung nach Kilger, in: Männel, Wolfgang (Hrsg.): Handbuch Kostenrechnung, Wiesbaden, S. 226-246.

Troßmann, Ernst (2013): Investition als Führungsentscheidung - Projektrechnungen für Controller, 2. Aufl., München.

Troßmann, Ernst/Baumeister, Alexander/Werkmeister, Clemens (2013): Fallstudien im Controlling Lösungsstrategien für die Praxis, 3. Aufl., München.

Vormbaum, Herbert (1966): Kalkulationsarten und Kalkulationsverfahren, 4. Aufl., Stuttgart 1977 (Erstauflage 1966).

Wagenhofer, Alfred (1992): Abweichungsanalysen bei der Erfolgskontrolle aus agency theoretischer Sicht, in: Betriebswirtschaftliche Forschung und Praxis, Jg. 44, S. 319-338.

Weber, Jürgen (2002): Logistikkostenrechnung: Kosten-, Leistungs- und Erlösinformationen zur erfolgsorientierten Steuerung der Logistik, Berlin etc.

Yermack, David (2015): Corporate Governance and Blockchains. Working Paper: NYU Stern School of Business and National Bureau of Economic Research, November 2015.

Ziegler, Hasso (1994): Neuorientierung des internen Rechnungswesens für das Unternehmens-Controlling im Hause Siemens, in: Zeitschrift für betriebswirtschaftliche Forschung, Jg. 46, S. $175-188$.

Zimmermann, Frank (2015): Gelebtes Green Controlling - bewertete Energieeffizienz in der Kostenträgerrechnung, in: rechnungswesen \& controlling, o. Jg., Heft 4, S. 6-8. 
Dieter Pfaff, Prof. Dr., Inhaber des Lehrstuhls Accounting im Institut für Betriebswirtschaftslehre der Universität Zürich

Anschrift: Universität Zürich, Affolternstrasse 56, 8050 Zürich, E-Mail: dieter.pfaff@business.uzh.ch, Homepage: www.business.uzh.ch

Ernst Troßmann, Prof. Dr., Inhaber des Lehrstuhls Controlling im Institut für Financial Management der Universität Hohenheim

Anschrift: Universität Hohenheim, Schloss Hohenheim 510 C, 70593 Stuttgart, E-mail: control@uni-hohenheim.de, Homepage: www.controlling.uni-hohenheim.de 